\title{
Revealing Drug Self-Associations into Nano-Entities
}

\author{
Marwa M. Dlim, ${ }^{\dagger}$ Fatma S. Shahout, ${ }^{\dagger}$ Marwa K. Khabir, Patrick P. Labonté, and Steven R. LaPlante*(i) \\ INRS, Institut Armand-Frappier, Université du Québec, 531, boul. des Prairies, Laval, Québec H7V 1B7, Canada
}

\section{Supporting Information}

ABSTRACT: The aqueous properties of the drugs Sorafenib, Lapatinib, Gefitinib, Fulvestrant, and Clofazimine were explored to monitor their tendency to self-associate. A combination of nuclear magnetic resonance, dynamics light scattering, and electron and confocal microscopies found that they tended to form large nano-entities having distinct types and sizes and were capable of entering cells. The combination of strategies employed serves to detect and reveal nano-entities along with their three-state equilibria and behaviors in buffers, media, and cells.

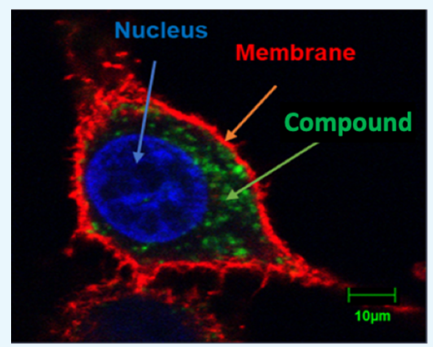

\section{INTRODUCTION}

The drug discovery community has recognized that the physicochemical attributes of compounds can somehow predispose them to many properties, ${ }^{1-9}$ so pharma workflows focus on prioritizing compound candidates that exhibit favorable properties and deprioritizing those that have undesirable properties. For this, extensive characterization efforts are undertaken.

For many reasons, these characterization efforts are executed and interpreted within the context that compounds in aqueous solvent behave predominantly as either single-molecules in solution or as a solid form such as precipitates. However, it is becoming more apparent that each compound exists in a unique three-phase equilibrium in solution between single lone-tumbling molecules, self-associated aggregates (nanoentities), and solid forms. Although this revised view recognizes the existence of this intermediate aggregate phase, it is becoming clear that drugs can form a wider range of selfassembled nano-entities than previously expected. ${ }^{10,12}$

To date, little is known about the full range of types and sizes of self-assemblies that drugs can adopt. There have been reports that some can form colloidal aggregates, whereas others can form much smaller multimers. ${ }^{10}$ One of the main issues for properly characterizing these nano-entities is insufficient detection strategies, which explains our poor knowledge of this phenomenon and the resultant properties. ${ }^{11-14}$ No single technology can detect the full range of nano-entities that can exist, but each technology has its advantages and limitations. For example, dynamics light scattering (DLS) and transmission electron microscopy (TEM) are sensitive to large colloidal assemblies (e.g., nanometer size) but are less optimal for small entities and mixtures. nuclear magnetic resonance (NMR) spectroscopy, on the other hand, is highly sensitive to small- to medium-sized aggregates (Ångstrom to subnanometer sizes). This technology can also be used to monitor large aggregates although it requires breaking the aggregates into smaller entities using detergents for detection purposes. Also, confocal laser scanning microscopy (CLSM) can be employed to monitor drugs in cells, but the compounds must be fluorescent and form sufficiently large assemblies. Other potential detection methods can also be used such as nephelometry, SPR, MST, DOSY NMR, and CPMG NMR.

Establishing appropriate detection strategies will be central for thoroughly correlating nano-entities with their respective properties. Impressive examples have already begun to emerge that demonstrate serious impact on drug discovery efforts. Compound and drug aggregates have been attributed to the observation of promiscuity and high incidences of falsepositives in high-throughput screens for lead discovery. ${ }^{13,14}$ They have also been implicated in affecting the efficacy of drugs in cell culture assays because of lack of cell membrane permeability. ${ }^{15}$ This has helped to explain the "bell-shaped" concentration response curves for formulated drugs. ${ }^{16}$ Furthermore, they have been responsible for giving rise to promiscuity in vitro, off-target pharmacology assays and toxicity alerts. ${ }^{10}$ Interestingly, aggregates have also been associated with beneficial attributes such as enhancing exceptional drug oral bioavailability. ${ }^{17}$ One can also envisage drug nano-entities as potential drug carriers or even delivery systems.

Here, we use several anticancer drugs (Sorafenib, Lapatinib, Gefitinib, and Fulvestrant) and an anti-leprosy drug (Clofazimine) as model systems to explore various techniques for monitoring their physicochemical solution behavior. We evaluate data from NMR, DLS, TEM, and CLSM to characterize the nano-entities formed and to probe the strengths and limitations of the methods. It should be kept in mind that the present study focuses on compounds that form the large colloidal aggregates. Studies involving the smaller nano-forms are referred to an early report and to forthcoming disclosures. ${ }^{10,12}$

Received: March 10, 2019

Accepted: May 7, 2019

Published: May 23, 2019 


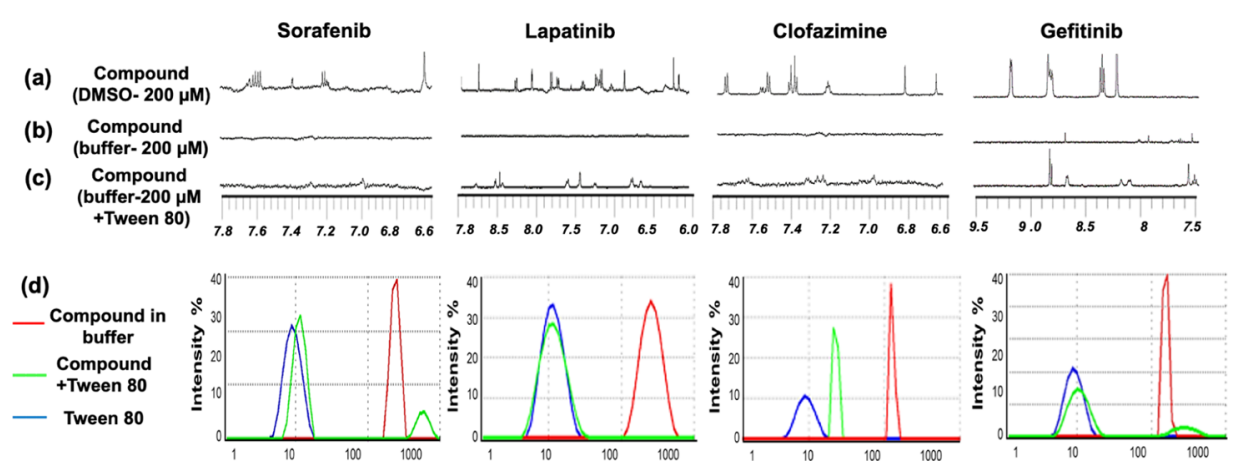

Figure 1. NMR spectra of four compounds. (a) Compounds in DMSO- $d_{6}$ at $200 \mu \mathrm{M}$, (b) compounds in buffer at $200 \mu \mathrm{M}$, (c) compounds in the presence of Tween 80, (d) DLS data for the four compounds. Both techniques involving buffer contained $50 \mathrm{mM}$ sodium phosphate, $100 \mathrm{mM}$ $\mathrm{NaCl}, 10 \% \mathrm{D}_{2} \mathrm{O}, \mathrm{pH} 7.4$ in the absence and presence of $0.025 \%(\mathrm{v} / \mathrm{v})$ Tween 80 for $24 \mathrm{~h}$.

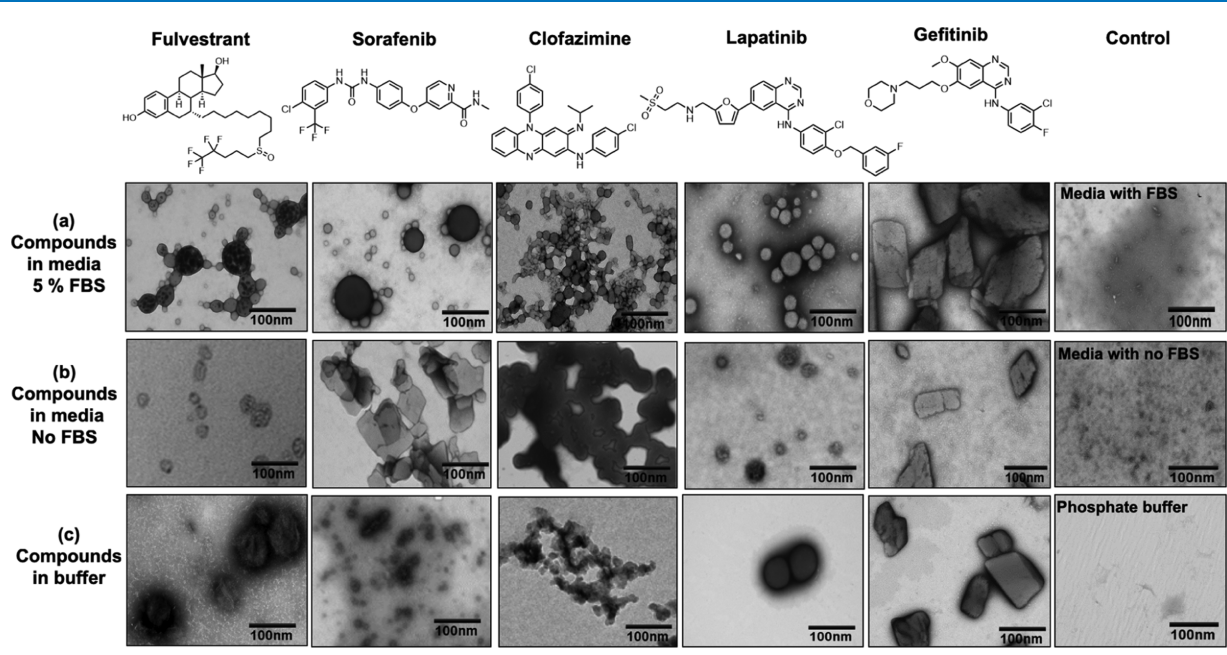

Figure 2. TEM images of four anticancer drugs (Fulvestrant, Sorafenib, Lapatinib, and Gefitinib), and an antileprosy drug (Clofazimine), (a) 50 $\mu \mathrm{M}$ of compounds incubated for $24 \mathrm{~h}$ in DMEM 5\% FBS, or (b) in DMEM with no FBS, or (c) in phosphate buffer pH 7.4. Bars represent $100 \mathrm{~nm}$.

\section{RESULT AND DISCUSSION}

A typical workflow practiced in the pharmaceutical industry is one where medicinal chemists synthesize new compounds based on design concepts intended to capture a range of intended favorable properties, for example, binding and specificity for a target protein, bioavailability, stability, and safety.

Medicinal chemists almost exclusively characterize their candidate drugs in organic solvents, then lyophilize, and expedite the powders or stock solutions to multiple other laboratories for a broad range of pharmaceutical tests where the compounds are dissolved in or diluted with aqueous media. However, drugs behave much differently in organic solvents as compared to aqueous media, and thus the above workflow introduces an important and uncharacterized disconnect. That is, no one along the workflow is responsible for monitoring a compound's aqueous behavior in solution for the aggregate phase-thus, the three-phase equilibrium systematically goes largely unexplored.

This is unfortunate because a simple and quick perusal of the ${ }^{1} \mathrm{H}$ NMR spectrum of a compound in buffer can easily begin to expose features of its three-state equilibrium. ${ }^{12}$ This is illustrated in (Figure 1) for the four compounds (Sorafenib, Lapatinib, Clofazimine, and Gefitinib). From $20 \mathrm{mM}$ stock solutions in DMSO- $d_{6}$, compounds were diluted in DMSO- $d_{6}$ solvent to $200 \mu \mathrm{M}$ concentration where it was noted that they dissolved well-clear solutions were observed with no precipitate. ${ }^{1} \mathrm{H}$ NMR spectra of the later samples $(200 \mu \mathrm{M})$ were then acquired, respectively, and shown in Figure 1a. This atomic view of hydrogen nuclei shows that all resonances are observable and sharp, as expected for compounds that behave as single lone-tumbling molecules in solution.

Samples of these compounds at $200 \mu \mathrm{M}$ in aqueous buffer were then prepared by placing aliquots of DMSO- $d_{6}$ stock solutions into aqueous buffer followed by gentle agitation. Some cloudiness or solid precipitate was noted, and so the samples were subjected to light centrifugation. The supernatant was then placed in NMR tubes and ${ }^{1} \mathrm{H}$ NMR spectra acquired. Figure $1 \mathrm{~b}$ shows that no NMR resonances were observed. It is possible that the compounds totally existed as a solid-state form and were removed by this latter manipulation. Even if some solid remained as a cloudiness, the resonances of solids are too broad to be observed by solution NMR. Another possible explanation would be that the compounds partitioned between precipitates and very large self-associated and soluble aggregates. The latter would have to tumble too slowly in solution which would also result in resonances that are too broad to be observed by solution NMR. Interestingly, we showed in a previous report that a simple trick of adding a detergent such as Triton or Tween to the samples induced the breakup of the large aggregates, resulting in faster tumbling lone molecules, which then gave rise to observable NMR resonances. To our surprise, the addition of detergent to the 
(a)

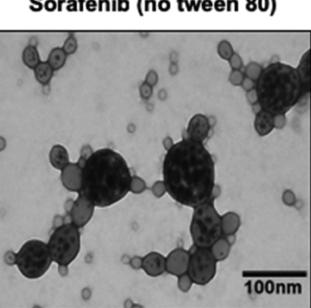

Sorafenib (+ tween 80 )

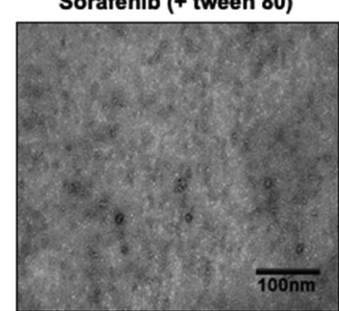

Lapatinib (no tween 80)

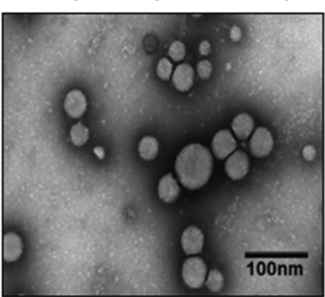

Lapatinib (+ tween 80)

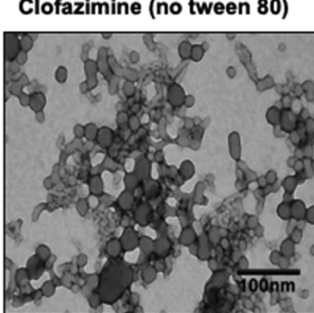

Clofazimine (+ tween 80)
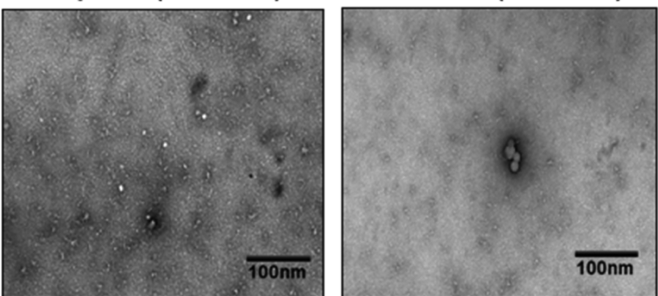

Figure 3. (a) TEM images of $50 \mu \mathrm{M}$ Sorafenib, Lapatinib, and Clofazimine incubated for $24 \mathrm{~h}$ at $37{ }^{\circ} \mathrm{C}$ in DMEM $5 \%$ FBS in the absence of $0.025 \%(\mathrm{v} / \mathrm{v})$ Tween 80, (b) in the presence of $0.025 \%(\mathrm{v} / \mathrm{v})$ Tween 80 . Bar represent $100 \mathrm{~nm}$.

Sorafenib sample did not give rise to sharp resonances (see Figure 1c), whereas sharp resonances did arise for Lapatinib, Clofazimine, and Gefitinib (see Figure 1c). The latter observations unequivocally report the existence of the large aggregates. However, the lack of resonances for Sorafenib demonstrates our assumptions and limited knowledge of aggregate types and how to manipulate and observe them by NMR.

Interestingly, DLS data acquired on the aqueous samples, after light centrifugation, clearly showed existence of the nanometer-sized aggregates (see Figure 1d). The DLS data of these samples after the subsequent addition of detergent report differential changes in aggregate sizes, which demonstrated the potential complementarity of NMR and DLS techniques. TEM also convincingly revealed the presence of very large aggregates in various media such as Dulbecco's modified Eagle's medium (DMEM) with 5\% fetal bovine serum (FBS) (see Figure 2a), cell culture media (DMEM) without (FBS) (see Figure 2b), and aqueous phosphate buffer (see Figure 2c). Similar experiments and conditions were also reported by others in which they found that the behaviors of the three anticancer drugs Lapatinib, Fulvestrant, and Sorafenib are consistent with the formation of colloidal aggregates in phosphate buffer and in cell culture media $10 \% \mathrm{FBS}$ for $24 \mathrm{~h}$ at $37^{\circ} \mathrm{C} .{ }^{15}$

First, it must be kept in mind that our samples were prepared by soaking the compound solutions with a carboncoated copper grid which is required for TEM observation purposes. Also, it is expected that lone-tumbling single molecules (tumbling radius on the single digit Ångstrom scale) would be invisible by TEM which is sensitive to species that have radii on the double-digit nanometer scale. Keeping these considerations in mind, a number of observations can nonetheless be made for characterizing these intriguing and large nano-entities observed (see Figure 2).

Interestingly, a comparison of the horizontal images along (Figure 2c) shows a variety of large aggregates for the drugs in buffer. Some are smaller such as that found for Clofazimine, whereas very large globs are noted for Fulvestrant, Sorafenib, and Lapatinib. A range of sizes are also noted. Gefitinib appears as a solid-like form. Changes in the aggregates are notable when the compounds are soaked in DMEM mediacompare Figure $2 \mathrm{a}$ with $2 \mathrm{~b}$. Likewise, dramatic changes are observed when comparing all three media conditions (see Figure $2 a-c)$. We then studied the effect of adding detergent to large nano-entities. The addition of detergents to samples suspected of forming large aggregates is a widely used strategy in many biochemical assays to reveal false-positive hits in screening campaigns.

One typically runs screening campaigns to identify lonetumbling compounds that inhibit a protein, but these assays are frequently contaminated with false-positive hits from compounds which form large aggregates and inhibit via nonstoichiometry and nonspecific means. Running follow-up validation screens typically involves the addition of a detergent, which presumably breaks up drug aggregates, and results in the loss of false-positive inhibition.

We thus explored the effect of the addition of detergents on the aggregates of the drugs studied here (see Figure $3 a$ ). The TEM images shown in Figure 3a for Sorafenib, Lapatinib, and Clofazimine (in the absence of detergent) clearly display large aggregates. Upon addition of $0.025 \%$ Tween 80 detergent, the TEM images in Figure $3 \mathrm{~b}$ show that these large aggregates have been seriously altered and disrupted. Taking the ${ }^{1} \mathrm{H}$ NMR experiments in Figure 1 into account, it is clear that addition of detergent breaks the aggregates into very small tumbling entities for Lapatinib and Clofazimine, which is consistent with the TEM changes in Figure $3 \mathrm{~b}$. However, small entities were not observed for Sorafenib upon addition of detergent, whereas the TEM data clearly shows disruption of the large aggregates. Therefore, the example of Sorafenib demonstrates that solubility is limited, or more likely here, there might be aggregate types that NMR and TEM simply cannot detect. This suggests that each compound can assume its unique fingerprint of self-assemblies, thus one must remain vigilant regarding assumptions and dogma in this field of study.

Two notable and contentious assumptions are that compound aggregates cannot exist in plasma nor be able to cross membranes to enter cells. ${ }^{18}$ The former assumption has often been rationalized, given that many drugs have been found to be highly serum-bound in vivo. ${ }^{19}$ Given this, the majority of a compound would be expected to be bound to serum proteins such as albumin, leaving compounds mostly unavailable to self-associate. This assumption is unfounded. In a previous study, involving an NMR aggregation test, it was 
clearly shown that aggregating compounds remained selfassociated in a range of pharmacology buffers, plasma, and blood. This would suggest that there is a significant affinity for self-association for some compounds. ${ }^{10}$ It has also recently been reported that Evans Blue forms colloids that adsorb albumin. ${ }^{15}$

Regarding these assumptions, we set out here to identify tools that can help determine if aggregating compounds can cross membranes and enter cells. Others assumed that aggregates are too large and cannot diffuse through cell membrane of live cells. ${ }^{16}$ For our study, we explored the use of CLSM. First, one must realize and consider that all the methods used here make observations at very distinct resolutions. NMR makes measurements at the atomic level or Ångstroms, whereas TEM and DLS resolve particles at the nanometer scale, and CLSM at the micrometer level. Thus, CLSM is the most appropriate method for observations at the cellular level. However, to render a compound observable by CLSM, it must have inherent fluorescence, so we were limited in the compounds that could be studied-thus, potential compounds were prescreened by a standard fluorescent microscope.

We began this study by the incubation of HeLa cells in the presence of Lapatinib at $50 \mu \mathrm{M}$ for $24 \mathrm{~h}$ and acquired CLSM views (see Figure 4a). Data from a range of other

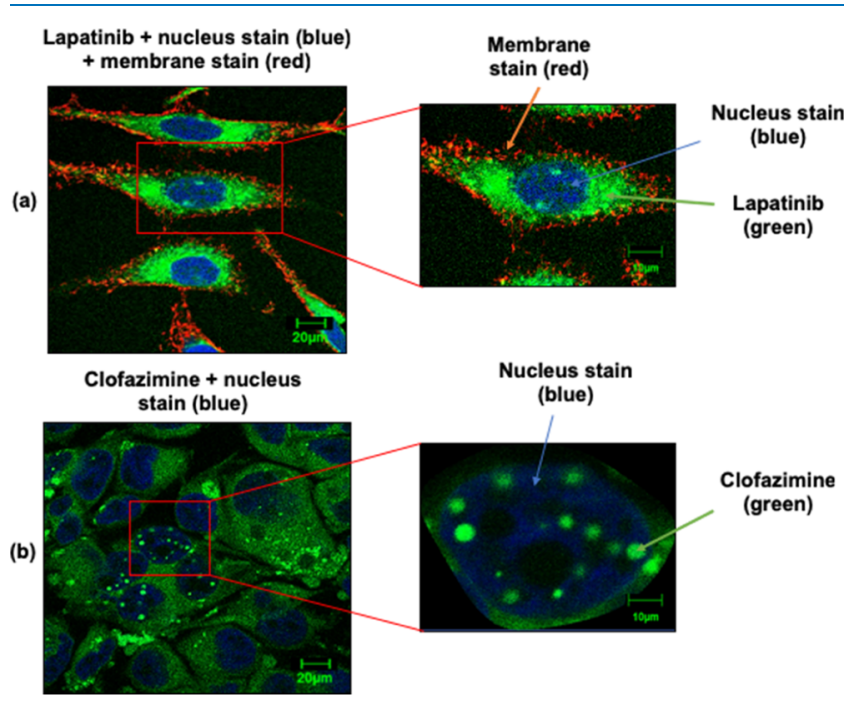

Figure 4. (a) Confocal images of HeLa cells incubated in the presence of $50 \mu \mathrm{M}$ Lapatinib for $24 \mathrm{~h}$. Alexa fluor WGA 555 was used to stain cell membranes and DRAQ5 to stain the nucleus. (b) Huh-7 cells incubated in the presence of $50 \mu \mathrm{M}$ Clofazimine for $24 \mathrm{~h}$. DAPI was used to stain the nucleus. Bar represents 20 and $10 \mu \mathrm{m}$. concentrations, conditions, and compounds are provided in the Supporting Information, along with a description of the procedures employed and experimental information. Figure $4 \mathrm{a}$ shows that the green fluorescent of Lapatinib indeed had entered the cell and appears mostly localized to the cytoplasm. Note that the cell membrane and nuclei can be visualized (see Figure $4 \mathrm{a}$ ) based on the red and blue dyes Alexa Fluor 555 conjugate of WGA and DRAQ5, respectively, which are wellknown markers. Interestingly, Lapatinib appears to be welldistributed within the cytoplasm given that green fluorescence is observed for all the cytoplasm.

We also measured the antiproliferative activities of aggregate form (no Tween) and monomer forms (+Tween) of Lapatinib on HeLa cells. Our study found that both forms exhibit similar antiproliferative activities suggesting that both forms are capable of entering HeLa cells (see Supporting Information, Figure S6). Note that we and others confirmed that $0.025 \%$ of Tween 80 was nontoxic (Supporting Information). ${ }^{15}$ In contrast, others found that the monomer form had significantly improved activity versus the aggregate form using MDA-MB231 cells, suggesting that the monomer form has better capability of entering cells as compared to the aggregate from. Thus, aggregate penetration is likely cell type-dependent.

Further CLSM studies were then focused on another compound Clofazimine, and distinct findings were observed. Clofazimine was incubated with Huh-7 cells for 1 hour (see Figure $4 b$ ). Although Figure $4 \mathrm{~b}$ shows a well-distributed green fluorescence within the cytoplasm, there are stronger compound signals arising from the nucleus. Note that the nuclei can be visualized (see Figure $4 \mathrm{~b}$ ) based on the blue dye DAPI, but the cell membrane cannot be easily distinguishable given that no Alexa Fluor 555 conjugate of WGA dye was added in this experiment because of signal interferences. In order to verify that the compound aggregates were occurring intra- and not extra-cellularly, care was taken to wash the cells after the incubation step. They were washed twice in phosphate-buffered saline (PBS) to remove any existing extracellular aggregates, and fresh DMEM 5\% FBS were added to the cells. ${ }^{20}$

It is also interesting that Clofazimine is considered as a lipophilic antibiotic which has very long pharmacokinetic halflife of up to 70 days. $^{20}$ It was noted that Clofazimine aggregates/accumulates in cells (in vitro) over several days, where it formed intracellular inclusions in the cytoplasm. Perhaps the long half-life is related to sequestering via aggregation. It was also reported that Clofazimine can be toxic as it induces changes to the mitochondria structure and function. Also, Clofazimine has reported to form stable complexes with DNA and transfer RNA, which resulted in spectral red shifts. ${ }^{21}$ In our study, no red shifts were observed
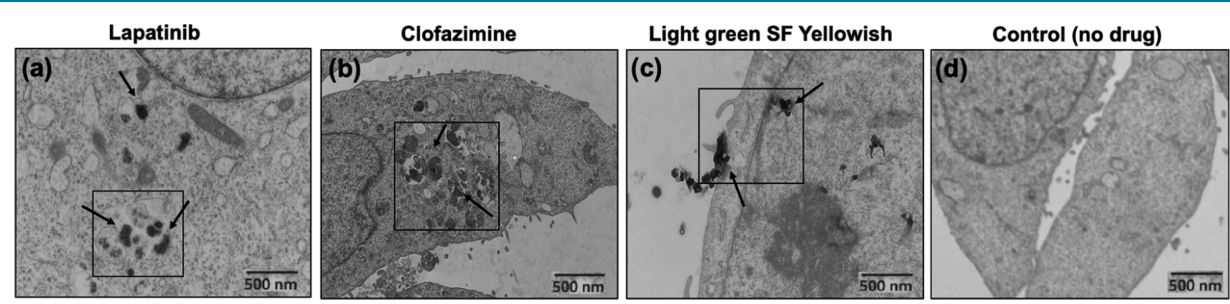

Figure 5. USEM images of HeLa cells incubated for $24 \mathrm{~h}$ in the presence of (a) $50 \mu \mathrm{M}$ Lapatinib, (b) $50 \mu \mathrm{M}$ Clofazimine, and (c) $50 \mu \mathrm{M}$ Light green SF yellowish, (d) represents control cells in the absence of drugs. Bar represent $500 \mathrm{~nm}$. 
and detections were made using the same green emission wavelengths in the nucleus and cytoplasm.

Our study also employed ultrathin section electron microscopy (USEM) to observe aggregates within cells but as a complementary technique with higher resolution at the nanometer scale. Figure 5 shows USEM images involving Clofazimine, Lapatinib, and Light green SF yellowish to probe these compounds in cells. Interestingly, distinct dark inclusions were noted in the presence of compounds. These inclusions appeared to resemble the size, number, and distribution to the drug inclusions observed by transmission light microscopy.

\section{CONCLUSIONS}

This work begins the process of evaluating tools for detecting large nano-entities. We found some strengths and weaknesses of a set of techniques, which nonetheless together have allowed us to reveal features of nano-entities from the atomic level to the micrometer scale. It was noted that these large nanoentities can adopt a variety of sizes and types that highly depend on the solution conditions. It was also confirmed that compounds that form nano-entities can indeed enter cells, and will certainly have properties (e.g., see ref 20).

However, it is still unclear where nano-entities can enter cells as the monomer form versus aggregate form. Perhaps this is cell and aggregate dependent. Others suggest that colloids do not, ${ }^{15,16}$ whereas we observe compounds that have colloidal forms and can enter cells. The exact mechanism of entry remains unknown.

Another important point is whether the compounds exist in cells as self-associated aggregates or subcellular localized with organelles. Perhaps both occur. The self-association into aggregates certainly helps to augment the observed fluorescence signal (see Figures 4 and $S 1-S 5$ ) as compared to a fluorescent compound Tartrazine that behave as lone-tumbling molecules and have low signal-to-noise (Figure S7). Certainly, colocalization also occurs. Figure S1 suggests that Lapatinib can colocalize with lysosomes. To better address the question of intracellular self-association, further studies are warranted involving the lack and addition of tween. Unfortunately, the toxicity of some compound studies here impeded such experiments.

It is our assessment that the scientific community has only begun to reveal this fascinating drug nano-world. First, a platform of techniques needs to be established, which will allow the scientific community to characterize nano-entities then establish their correlation with salient properties. For example, it has already been established that large-nano-entities can be correlated with compounds high bioavailability, promiscuity, false-positives in screens, and so forth. Perhaps a better understanding of nano-entities can help minimize drug side effects, promote safer compounds to the clinic, or to serve as drug delivery systems. For example, the corresponding author has used the detection of aggregates to deprioritize promiscuous drug candidates and promote selective compounds for the clinic. ${ }^{12,22,23}$

\section{EXPERIMENTAL SECTION}

Compounds (Drugs and Dyes). Drugs and dyes used in this study were obtained from commercial vendors. CAS numbers are as following: Sorafenib (284461-73-0) from Synchem, Inc.; Fulvestrant (129453-61-8) from Sigma; Lapatinib (388082-78-8) from Larid Road; Clofazimine
(2030-63-9) from Sigma; Tartrazine (1934-21-0), and Light green SF yellowish (5141-20-8) from Alfa Aesar. Alexa fluor WGA555 and Prolong Diamond Anti-fade with DAPI was purchased from Thermo Fisher Scientific.

Transmission Electron Microscopy. Compounds were diluted in $50 \mathrm{mM}$ sodium phosphate $\mathrm{pH} 7.4$ and in DMEM 5\% FBS. Next, $100 \mu \mathrm{L}$ of the samples were transferred into a 240 $\mu \mathrm{L}$ Airfuge tube. A carbon-coated copper grid was inserted into the bottom of the Airfuge tube with fine tweezers and centrifuged for $5 \mathrm{~min}$ at 20 psi. The carbon grid was gently removed with tweezers and washed with distilled water, and the carbon grid was negatively stained with $3 \%$ of phosphotungstic acid (PTA-3). The grid was removed, blotted, and dried with a bibulous paper, then examined by transmission electron microscope (Hitachi H-7100). The photographs were processed with the digital camera AMT version 600.147.

Cell Culture. HeLa and Huh-7 cell lines were maintained in DMEM supplemented with $5 \%$ of FBS albumin and in $1 \%$ penicillin/streptomycin.

Confocal Laser Scanning Microscopy. CLSM was employed to observe the self-aggregation of compounds within the cells. HeLa and Huh-7 cells were grown on glass coverslips in 24 well plates and cultured overnight in DMEM (5\% FBS, $1 \%$ penicillin/streptomycin) at $37{ }^{\circ} \mathrm{C}$ in $5 \% \mathrm{CO}_{2}$. Cell culture media was removed and washed twice with PBS (50 mM sodium phosphate, $100 \mathrm{mM}$ sodium chloride). Compounds at the given concentrations were added to the wells. After 1, 2, 4, 6 , and $24 \mathrm{~h}$ of incubation, the cells were washed twice with $1 \times$ PBS (50 mM sodium phosphate, $100 \mathrm{mM}$ sodium chloride) and treated with Alexa Fluor WGA555 for membrane staining. The cells were then fixed in $4 \%$ paraformaldehyde solution for 10 minutes and washed twice with PBS $1 \times$. Further, the coverslips were mounted on a prolong diamond antifade with DAPI nucleus staining for Clofazimine and Light green SF yellowish and DRAQ-5 for Lapatinib. The cells were imaged by a Zeiss CLSM-780 confocal microscopy (ZEISS, Jena, Germany) on an Olympus FV1000 at $60 \times$ magnification, using the excitation and emission wavelengths for DAPI, excitation was at $405 \mathrm{~nm}$ and emission at $460 \mathrm{~nm}$; for Alexa Fluor WGA555, excitation and emission were at 520 and $550 \mathrm{~nm}$, respectively. Compound excitation and emission information are as follows: Clofazimine excitation (460-495 nm) and emission (515-550 $\mathrm{nm})$, Lapatinib excitation $(405 \mathrm{~nm})$ and emission $460 \mathrm{~nm}$ ), and Light green SF yellowish excitation $(405 \mathrm{~nm})$ and emission $(460 \mathrm{~nm})$.

Cell Proliferation Assay and in Vitro Cytotoxicity Study (MTT Assay). The cytotoxicity of the panel of compounds and DMSO were evaluated in vitro using MTT assays. HeLa and Huh-7 cells were plated in 96-well plates and cultured overnight in DMEM 5\% FBS, in the absence or presence of compounds. After removing the culture medium, various concentrations of the compounds added to the cells, and incubated for $24 \mathrm{~h}$. The medium in each well was then aspirated and discarded. Next, $10 \mu \mathrm{L}$ of $5 \mathrm{mg} / \mathrm{mL}$ MTT solution 3-(4,5-dimethylthiazol-2,5-diphenyl tetrazolium bromide), was added to each well and incubated for 2 to $4 \mathrm{~h}$. Following incubation, the medium was replaced with $150 \mu \mathrm{L}$ DMSO solution. After $15 \mathrm{~min}$, the optical densities at $570 \mathrm{~nm}$ were measured by spectrophotometer.

Dynamic Light Scattering. Compounds were measured in DMEM 5\% FBS or phosphate buffer, $\mathrm{pH}$ 7.4. Measurements were performed by polystyrol/polystyrene $10 \times 10 \times 45 \mathrm{~mm}$ 
cuvettes, utilizing the Zetasizer Nano ZS (Malvern), version 1.7 , with a $60 \mathrm{~mW}$ laser operating at $830 \mathrm{~nm}$ and a detector angle of $158^{\circ}$. All samples were centrifuged before analysis performed in triplicate at $25{ }^{\circ} \mathrm{C}$ and the data were acquired using the Dynamics software. Compounds were at $50 \mu \mathrm{M}$.

Nuclear Magnetic Resonance. Compounds were prepared from $20 \mathrm{mM}$ DMSO stock solutions into buffer consisting of $50 \mathrm{mM}$ sodium phosphate $\mathrm{pH} 7.4$ and $10 \%$ $\mathrm{D}_{2} \mathrm{O}$. DMSO samples were prepared by diluting the stock samples. NMR data was acquired on a Bruker $600 \mathrm{MHz}$ NMR spectrometer equipped with a helium cryoprobe. ${ }^{10,12}$

Thin-Section Electron Microscopy. The cells were plated at $1 \times 10^{5}$ cells/well and incubated in the presence of $50 \mu \mathrm{M}$ of all compounds for $24 \mathrm{~h}$. The medium was then aspirated, and the cells washed two times with $1 \times$ PBS. The cells were then fixed with glutaraldehyde $(2.5 \%$ in $0.1 \mathrm{M}$ cacodylate buffer or phosphate-buffered saline, overnight) and again washed $2 \times$ with PBS. Next, the cells were collected and centrifuged at $1000 \mathrm{~g}$ for $10 \mathrm{~min}$. The fixed-cell pellets were resuspended in a freshly prepared solution $1.3 \%(\mathrm{w} / \mathrm{v})$ osmium tetroxide in a colliding buffer for $1-2 \mathrm{~h}$ and then dehydrated by successive washes with $25,50,75$, and $95 \%$ solutions of acetone in water (15-30 min each). This was followed by two changes of pure acetone incubated for $30 \mathrm{~min}$ each. The cell pellets were then resuspended in SPURR acetone (1:1) and incubated for 16$18 \mathrm{~h}$ at room temperature. The cells were cut into small pieces and placed in BEEM capsules to capacity. The capsules were incubated at $600-650{ }^{\circ} \mathrm{C}$ for the polymerization reaction to occur. The final stage involved cutting the embedded cells into ultrathin sections and placing the sections on a carbon-covered copper 200-mesh grid. The grids were then stained with 50\% ethanol for 20-25 min. Examination of the sections was performed using an electron microscope (Hitachi H-7100), and the photographs were processed using the digital camera AMT, version 600.147.

\section{ASSOCIATED CONTENT}

\section{S Supporting Information}

The Supporting Information is available free of charge on the ACS Publications website at DOI: 10.1021/acsomega.9b00667.

Experimental section, additional data, and confocal and electron microscopy images (PDF)

\section{AUTHOR INFORMATION}

\section{Corresponding Author}

*E-mail: steven.laplante@iaf.inrs.ca. Phone: +1-514-914-8501. ORCID $\odot$

Steven R. LaPlante: 0000-0002-9394-6553

\section{Author Contributions}

${ }^{\dagger}$ M.M.D. and F.S.S. contributed equally to this work and should both be considered as first authors.

\section{Notes}

The authors declare no competing financial interest.

\section{ACKNOWLEDGMENTS}

The authors wish to acknowledge technical support from J. Tremblay (confocal microscopy), Sami Alsabri (assay) and from M. Letarte and A. Nakamura (electron microscopy). This work was supported by the Libyan Government, NSERC and NMX Research and Solutions Inc.

\section{ABBREVIATIONS}

NMR, nuclear magnetic resonance; DLS, dynamic light scattering; TEM, transmission electron microscope; TPSA, total polar surface area; CLSM, confocal laser scanning microscopy; DMEM, Dulbecco's modified Eagle's medium; FBS, fetal bovine serum; BPS, phosphate-buffered saline; USEM, ultra-thin section electron microscopy

\section{REFERENCES}

(1) Leeson, P. D.; Springthorpe, B. The influence of drug-like concepts on decision-making in medicinal chemistry. Nat. Rev. Drug Discovery 2007, 6, 881.

(2) Lipinski, C. A.; Lombardo, F.; Dominy, B. W.; Feeney, P. J. Experimental and computational approaches to estimate solubility and permeability in drug discovery and development settings. Adv. Drug Delivery Rev 1997, 23, 3.

(3) Lipinski, C. A. Lead-and drug-like compounds: the rule-of-five revolution. Drug Discovery Today: Technol. 2004, 1, 337.

(4) Vieth, M.; Siegel, M. G.; Higgs, R. E.; Watson, I. A.; Robertson, D. H.; Savin, K. A.; Durst, G. L.; Hipskind, P. A. Characteristic physical properties and structural fragments of marketed oral drugs. J. Med. Chem. 2004, 47, 224.

(5) Proudfoot, J. R. The evolution of synthetic oral drug properties. Bioorg. Med. Chem. Lett. 2005, 15, 1087.

(6) Morphy, R. The influence of target family and functional activity on the physicochemical properties of pre-clinical compounds. J. Med. Chem. 2006, 49, 2969.

(7) Van De Waterbeemd, H.; Smith, D. A.; Beaumont, K.; Walker, D. K. Property-based design: optimization of drug absorption and pharmacokinetics. J. Med. Chem. 2001, 44, 1313.

(8) Cronin, M. D.; Mark, T. The role of hydrophobicity in toxicity prediction. Curr. Comput.-Aided Drug Des. 2006, 2, 405.

(9) Meanwell, N. A. Improving drug candidates by design: a focus on physicochemical properties as a means of improving compound disposition and safety. Chem. Res. Toxicol. 2011, 24, 1420.

(10) LaPlante, S. R.; Aubry, N.; Bolger, G.; Bonneau, P.; Carson, R.; Coulombe, R.; Sturino, C.; Beaulieu, P. L. Monitoring drug selfaggregation and potential for promiscuity in off-target in vitro pharmacology screens by a practical NMR strategy. J. Med. Chem. 2013, 56, 7073-7083.

(11) Hughes, J. D.; Blagg, J.; Price, D. A.; Bailey, S.; DeCrescenzo, G. A.; Devraj, R. V.; Ellsworth, E.; Fobian, Y. M.; Gibbs, M. E.; Gilles, R. W.; Greene, N.; Huang, E.; Krieger-Burke, T.; Loesel, J.; Wager, T.; Whiteley, L.; Zhang, Y. Physiochemical drug properties associated with in vivo toxicological outcomes. Bioorg. Med. Chem. Lett. 2008, 18, $4872-4875$.

(12) LaPlante, S. R.; Carson, R.; Gillard, J.; Aubry, N.; Coulombe, R.; Bordeleau, S.; Bonneau, P.; Little, M.; O'Meara, J.; Beaulieu, P. L. Compound aggregation in drug discovery: implementing a practical NMR assay for medicinal chemists. J. Med. Chem. 2013, 56, 51425150.

(13) Seidler, J.; McGovern, S. L.; Doman, T. N.; Shoichet, B. K. Identification and prediction of promiscuous aggregating inhibitors among known drugs. J. Med. Chem. 2003, 46, 4477-4486.

(14) Feng, B. Y.; Simeonov, A.; Jadhav, A.; Babaoglu, K.; Inglese, J.; Shoichet, B. K.; Austin, C. P. A high-throughput screen for aggregation-based inhibition in a large compound library. J. Med. Chem. 2007, 50, 2385-2390.

(15) Owen, S. C.; Doak, A. K.; Wassam, P.; Shoichet, M. S.; Shoichet, B. K. Colloidal Aggregation Affects the Efficacy of Anticancer Drugs in Cell Culture. ACS Chem. Biol. 2012, 7, 14291435.

(16) Owen, S. C.; Doak, A. K.; Ganesh, A. N.; Nedyalkova, L.; McLaughlin, C. K.; Shoichet, B. K.; Shoichet, M. S. Colloidal Drug Formulations Can Explain "Bell-Shaped" Concentration-Response Curves. ACS Chem. Biol. 2014, 9, 777-784.

(17) Frenkel, Y. V.; Clark, A. D.; Das, K.; Wang, Y.-H.; Lewi, P. J.; Janssen, P. A. J.; Arnold, E. Concentration and $\mathrm{pH}$ dependent 
aggregation of hydrophobic drug molecules and relevance to oral bioavailability. J. Med. Chem. 2005, 48, 1974.

(18) Ganesh, A. N.; Donders, E. N.; Shoichet, B. K.; Shoichet, M. S. Colloidal aggregation: From screening nuisance to formulation nuance. Nano Today 2018, 19, 188-200.

(19) Lu, J.; Owen, S. C.; Shoichet, M. S. Stability of self-assembled polymeric micelles in serum. Macromolecules 2011, 44, 6002.

(20) Baik, J.; Rosania, G. R. Molecular imaging of intracellular drug-membrane aggregate formation. Mol. Pharm. 2011, 8, 1742.

(21) Morrison, N. E.; Marley, G. M. Clofazimine binding studies with deoxyribonucleic acid. Int J Lepr Other Mycobact Dis 1976, 44, 475-481.

(22) Beaulieu, P. L.; Bolger, G.; Deon, D.; Duplessis, M.; Fazal, G.; Gagnon, A.; Garneau, M.; LaPlante, S.; Stammers, T.; Kukolj, G.; Duan, J. Multi-parameter optimization of aza-follow-ups to BI 207524, a thumb pocket $1 \mathrm{HCV}$ NS5B polymerase inhibitor. Part 2: Impact of lipophilicity on promiscuity and in vivo toxicity. Bioorg. Med. Chem. Lett. 2015, 25, 1140.

(23) LaPlante, S. R.; Bös, M.; Brochu, C.; Chabot, C.; Coulombe, R.; Gillard, J. R.; Jakalian, A.; Poirier, M.; Rancourt, J.; Stammers, T.; Thavonekham, B.; Beaulieu, P. L.; Kukolj, G.; Tsantrizos, Y. S. Conformation-based restrictions and scaffold replacements in the design of hepatitis $\mathrm{C}$ virus polymerase inhibitors: discovery of deleobuvir (BI 207127). J. Med. Chem. 2013, 57, 1845. 